\title{
Phase Diagram of a Loop on the Square Lattice
}

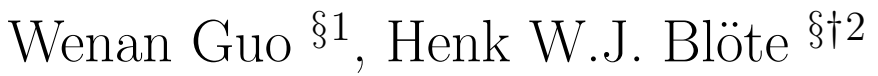 \\ and Bernard Nienhuis \\ $\S$ Faculty of Applied Science, P.O. Box 5046, 2600 GA Delft, The \\ Netherlands \\ † Instituut Lorentz, Universiteit Leiden, Niels Bohrweg 2, Postbus 9506, \\ 2300 RA Leiden, The Netherlands \\ $\ddagger$ Instituut voor Theoretische Fysica, Universiteit van Amsterdam, \\ Valckenierstraat 65, 1018 XE Amsterdam, The Netherlands
}

\begin{abstract}
The phase diagram of the $\mathrm{O}(n)$ model, in particular the special case $n=0$, is studied by means of transfer-matrix calculations on the loop representation of the $\mathrm{O}(n)$ model. The model is defined on the square lattice; the loops are allowed to collide at the lattice vertices, but not to intersect. The loop model contains three variable parameters that determine the loop density or temperature, the energy of a bend in a loop, and the interaction energy of colliding loop segments. A finitesize analysis of the transfer-matrix results yields the phase diagram
\end{abstract}

\footnotetext{
${ }^{1}$ present address: Physics Department, Beijing Normal University, Beijing 100875, P.

R. China

${ }^{2}$ e-mail: bloete@tn.tudelft.nl
} 
in a special plane of the parameter space. These results confirm the existence of a multicritical point and an Ising-like critical line in the low-temperature $\mathrm{O}(n)$ phase.

Keywords: $\mathrm{O}(n)$ model; Polymers; Phase diagram. 


\section{Introduction}

The $\mathrm{O}(n)$ model is originally defined as a model of $n$-component spins, with $\mathrm{O}(n)$ symmetric interactions. This model appears to be meaningful only when $n$ is a positive integer. However, a mapping [1] has been found on a model of closed loops in which $n$ appears as the Boltzmann weight associated with each loop: hence $n$ has become a continuously variable parameter. The critical point, which is characterized by e.g. the divergence of the susceptibility in the spin representation, corresponds with a diverging loop size in the loop model. The latter model, in particular in two dimensions, is accessible by exact and numerical analyses, and is thus also relevant for the exploration of the $\mathrm{O}(n)$ spin model. Indeed, a wealth of results have been found for the $\mathrm{O}(n)$ loop model. For instance, an exact critical line was found for an $\mathrm{O}(n)$ model on the honeycomb lattice, as well as the associated thermal and magnetic exponents [2, 3, 4, 5]. The critical line covers the interval $-2 \leq n \leq 2$ of the loop model and thus applies to $n=1$ and $n=2$ in the spin model. Another special case of the $\mathrm{O}(n)$ loop model is $n=0$, where the weight of a loop vanishes. This does not mean that the system is empty: the $\mathrm{O}(0)$ model may be formulated in terms of the derivative of the $\mathrm{O}(n)$ partition function to $n$, taken at $n=0$ so that one loop remains, finite or infinite. Thus the nonintersecting $\mathrm{O}(0)$ loop model can be applied to describe self-avoiding walks (SAW's) or polymers. Furthermore the $n=-2$ case corresponds to random walks without excluded-volume interactions [6, 7].

More complicated phase behavior is observed when attractive interactions between the loop segments are introduced. As long as these attractions are sufficiently small, the $\mathrm{O}(0)$ ordering transition remains in the same universality class. But when the interactions reach a certain threshold a tricritical point appears, called the theta point [8], and at even stronger attractions the phase transition turns first order. While exact information is scarce in the temperature versus attraction phase diagram, a numerical analysis [9] confirms the picture sketched here.

This tricritical theta point was first found [8] for a loop model with vacancies on the honeycomb lattice. The same type of transition, describing a collapsing polymer, was found for the square lattice $\mathrm{O}(0)$ loop model, namely on branch 0 of Ref. [10]. The latter model did, in addition to a temperature variable and an attraction between loop segments, also contain a parameter that distinguishes between straight loop segments and 90 degree bends. The results obtained for the square lattice included a generalization [10] of the 
theta transition to values $n \neq 0$. Remarkably, the enlarged parameter space of three parameters (not counting $n$ ) appears to contain also a different type of collapse transition [10, 11] for $n=0$. Like the theta point found on the square lattice, it generalizes to other values of $n$ (branch 3 of Ref. [10]).

To understand this second collapse mechanism, it may help to identify straight loop segments in terms of Ising-like degrees of freedom in the $\mathrm{O}(n)$ model [10, 9]. When the straight loop segments are suppressed, Ising-like order appears. Thus the newly observed multicritical point has been identified as the point along the $\mathrm{O}(n)$ critical line where the Ising degrees of freedom become critical. These new effects are a consequence of the underlying lattice structure and are thus not generally expected in a model of free polymers. However, they could be present in the case of a polymer influenced by a periodic potential caused by a crystalline substrate.

The freezing of these Ising degrees of freedom does not only occur on the line of phase transitions, but is also expected in the low-temperature $\mathrm{O}(n)$ phase. Thus an Ising-like transition would extend from the multicritical point into the dense phase of the loop model. Indeed, the results reported in Ref. [10] include a branch of critical points where the critical exponents reflect both the Ising and the low-temperature $\mathrm{O}(n)$ universal critical behavior.

These observations have been interpreted in terms of a conjectured and qualitative phase diagram [10]. In the present paper we confirm the validity of this phase diagram, including the predicted Ising line, by means of finitesize scaling and a transfer-matrix technique. Our investigations concern the $n=0$ case of the $\mathrm{O}(n)$ model on the square lattice as defined in Ref. [10. We briefly recall the definition of the partition function

$$
Z_{\text {loop }}=\sum_{\mathcal{L}} n^{N_{l}} x^{N_{x}} y^{N_{y}} z^{N_{z}}
$$

which depends on three vertex weights $x, y$, and $z$, associated with a 90degree bend of a loop segment, a straight loop segment, and a vertex visited by two 90-degree bends: a collision. Intersections are forbidden and a weight 1 is assigned to an empty vertex (not visited by a loop). The exponents $N_{x}$, $N_{y}$ and $N_{z}$ denote the total number of vertices of the type indicated. The number of closed loops is $N_{l}$, and thus, after differentiation with respect to $n$, only loop configurations $\mathcal{L}$ with $N_{l}=1$ contribute to the partition function of the $n=0$ model. When the weight $y$ of the straight loop segments is set to 0 , we freeze the Ising-like degrees of freedom mentioned above. Then the model reduces to that investigated in Ref. [9] and displays a theta point. 
The special character of the $y=0$ case also becomes apparent from the fact that the model, at least for even system sizes, is symmetric with respect to a change of sign of $y$. The point $y=0$ is special on the basis of this symmetry. In this paper we explore nonzero values of $y$ and restrict ourselves to the $x=y$ plane. The weight $x=y$ plays the role of a temperature-like parameter (at least when $z$ is small) and drives the SAW-type transition between the vacuum and the dense phase. The role of the parameter $z$ is to adjust the attractive interaction between loop segments.

The rest of this paper is composed as follows. In Section 2 we summarize the numerical procedures and in Section 3 we present the results and discuss the phase diagram thus obtained.

\section{Numerical procedures}

We consider the square $\mathrm{O}(0)$ model, wrapped on an infinitely long cylinder of circumference $L$, the finite size of the system, which is measured in elementary lattice spacings. One can derive quantities of interest, such as the free energy and correlation lengths, from the eigenvalues of the transfer matrix. For this purpose we make use of the construction of the $\mathrm{O}(n)$ loop model transfer matrix given in Ref. [10].

The relevant eigenvalues of the transfer matrix are obtained by a method explained e.g. in Ref. [12]. This method is based on iterative multiplication of a vector by the transfer matrix. Even when $n=0$ this procedure generates non-empty configurations containing a part of a loop. Closure of such a loop leads to a zero contribution to the resulting vector, but there will always be contributions due to configurations in which the loop is not (yet) closed. These configurations contribute to the eigenvalues of the transfer matrix. Therefore the transfer matrix allows the exploration of non-empty configurations of the $\mathrm{O}(0)$ model.

The $\mathrm{O}(n)$ spin-spin correlation function can simply be translated into the language of the $\mathrm{O}(n)$ loop model, as the ratio $Z^{\prime} / Z$, where $Z$ is the partition sum of the loop model and $Z^{\prime}$ a similar sum, but restricted to loop configurations that include a non-closed loop segment connecting the two correlated spins. For spins separated along the length direction of the cylinder, we may obtain $Z^{\prime}$ from the 'odd transfer matrix' that describes configurations which include such an additional single segment [10]. The

partition zum $Z$ is obtained from the 'even transfer matrix' that allows only 
configurations in which loop segments are pairwise connected at the end of the cylinder. This is a convenient method to derive the magnetic correlation length and its associated scaling dimension. For further details see Ref. [10].

The relation between the magnetic correlation length $\xi_{h}$, the largest eigen-

value $\Lambda_{L}^{(0)}$ of the even transfer matrix and the largest eigenvalue $\Lambda_{L}^{(1)}$ of the odd transfer matrix is

$$
\xi_{h}(L)=1 / \ln \left(\Lambda_{L}^{(0)} / \Lambda_{L}^{(1)}\right)
$$

The finite-size scaling behavior of $\xi_{h}$ and the principle of the 'phenomenological renormalization' [13] of $\xi_{h}$ provide a method to explore the phase diagram of the model. For this purpose we define the 'scaled gap' as $X_{h}(t, L)=$ $L / 2 \pi \xi_{h}(t, L)$. Apart from the finite size $L$, it depends on a temperature-like scaling field $t$. More than one scaling field can play a role but we consider only one field at a time.

For conformally invariant models, the scaled gaps $X_{h}(0, L)$ tend to the magnetic scaling dimension $X_{h}$ for $L \rightarrow \infty$ [14]. For nonzero but small $t$, we expect finite-size scaling behavior according to

$$
X_{h}(t, L)=X_{h}+1 /(2 \pi) t L^{y t}\left[d \xi_{h}^{-1}(t, 1) / d t\right]_{t=0}+\cdots
$$

where $y_{t}$ the renormalization exponent associated with $t$. If the temperaturelike field is irrelevant $\left(y_{t}<0\right), X_{h}(t, L)$ converges to $X_{h}$ with increasing $L$. If it is relevant, $X_{h}(t, L)$ diverges but for small $t$ Eq. (3) may still give a good description of the finite-size data within the range of available $L$ values. This formula serves well for the analysis of the finite-size results $X_{h}(t, L)$ in terms of the phase diagram.

\section{Transfer matrix analysis}

We restrict our parameter space by setting $x=y$. A preliminary scan of the phase diagram in the resulting plane, based on the leading eigenvalues and eigenvectors for small $L$, confirmed the existence of three distinct phases:

1. A high-temperature or vacuum phase for small $x$ and $z$. It corresponds with the paramagnetic phase in the $\mathrm{O}(n)$ spin language. The vacuum state is separated by a gap from non-empty states.

2. A moderately dense phase for large $x$ but not too large $z$. Type- $y$ vertices are abundant, suggesting Ising disorder as described in Ref. [10]. This is the gapless low-temperature phase of the $\mathrm{O}(n)$ spin model. 
3. At sufficiently large $z$ we observe an even denser phase, dominated by type- $z$ vertices. Type- $y$ vertices are scarce, suggesting Ising order. This phase is of the low-temperature $\mathrm{O}(n)$ type, but there exists a gap between the leading eigenvalues of the even and odd transfer matrices that does not vanish when $L \rightarrow \infty$. This is because, on lattices with even $L$, loop configurations composed of only type- $z$ vertices, occur only in the case of the even transfer matrix. The odd transfer matrix is unable to cover an even $L$ lattice completely with type- $z$ vertices.

In order to locate lines of phase transitions separating these three regions, we set $z$ at some fixed value, and adjust $x$ to its transition value $x_{c}$. We rewrite Eq. (3) as a function of $x$, where we may e.g. take $t=x-x_{c}$ in first order to represent the relevant temperature field that vanishes at the transition. This leads to

$$
X_{h}(x, L)=X_{h}(x, L+2)
$$

where we neglect corrections due to irrelevant fields. Thus numerical solution of $x$ of Eq. (4) yields an estimate of $x_{c}$. Solutions were obtained for even system sizes $L=2 \cdots 10$ in Eq. (田). We ignored the odd systems because they display additional effects due to odd-even alternation.

Figures 1 and 2 illustrate how these $x_{c}$ estimates are obtained. They show the scaled gaps for various system sizes as a function of $x$, for $z=0.3$ (Fig. 11) and for $z=0.8$ (Fig. 2). Intersections of the curves for successive values of $L$ yield estimates of $x_{c}$. The intersections do not coincide as a consequence of corrections to scaling, but seem to converge well with increasing size $L$. The expected corrections behave as negative powers of $L$ and extrapolation of the $x_{c}$ estimates was done accordingly. The results are shown in Fig. 3 .

Another way to investigate the phase diagram is to fix $x=y$ at some value and to vary $z$. For small values of $x$ the leading eigenvalues of the even transfer matrix display intersections, leading to a kink in the free energy when $z$ is increased: the first-order phase transition between the vacuum phase and the dense phase, as already expected in Ref. [10]. The intersections provide a convenient way to solve for the location of the first-order line: one just solves for the value of $z$ where the two largest eigenvalues become degenerate. This procedure leads to results that seem to converge well; they are included in Fig. 3. The data shown in this figure are in a full (but, of course, only qualitative) agreement with the conjectured phase diagram of Ref. [10]. 
The solutions of Eq. (4) do not only yield estimates of the critical point, but also for the corresponding magnetic scaling dimension $X_{h}$ which relates to the exponent $\eta$ describing the decay of the magnetic correlation function as $\eta=2 X_{h}$. This allows us to verify the expected universal behavior along the critical lines. Indeed, as is already illustrated in Fig. 1, the finite-size estimates of $X_{h}$ for the critical line at $z<0.4$ are in a good agreement with the exactly known value $X_{h}=5 / 48=0.104166 \cdots$ for the ordinary $\mathrm{O}(0)$ or SAW critical point. Furthermore, for $z>0.6$ the $X_{h}$ estimates seem to converge well to the expected value [10] $X_{h}=-1 / 16$ for branch 4 , which is the sum of the low-temperature magnetic exponent $(-3 / 16)$ and the Ising exponent (1/8). The crossover between these different ranges of $z$ appears to take place near $z=0.54$. At this value, the $X_{h}$ estimates behave in a way consistent with $X_{h}=-5 / 48$ as found for the $n=0$ point of branch 3 [11].

These results confirm that the unusual universal behavior reported for branches 3 and 4 [10] is not restricted to the special points that admit exact solutions, but apply to larger parameter spaces, at least for $n=0$. In the $x=y$ plane we locate one point corresponding with branch 3 , and a line of points that display branch- 4 like behavior. Since the $n=0$ points of branches 3 and 4 do not lie in the $x=y$ plane, it appears that the universality class of branch 3 covers a 1-dimensional manifold of the $x, y, z$ parameter space, and that the universality class of branch 4 covers a 2-dimensional manifold. It seems reasonable to expect that a similar picture exists for $n \neq 0$, at least for a range of $n$ values. This will be the subject of further investigations.

The calculations reported here are modest in size: they required only a few hours of CPU time on a Silicon Graphics workstation and a few hours on a personal computer.

Acknowledgements: This research is supported in part by the FOM ('Stichting voor Fundamenteel Onderzoek der Materie') which is financially supported by the NWO ('Nederlandse Organisatie voor Wetenschappelijk Onderzoek').

\section{References}

[1] P.G. de Gennes, Phys. Lett. A 38339 (1972).

[2] B. Nienhuis, Phys. Rev. Lett. 491062 (1982).

[3] R.J. Baxter, J. Phys. A 192821 (1986); J. Phys. A 205241 (1987). 
[4] M.T. Batchelor and H.W.J. Blöte, Phys. Rev. Lett. 61138 (1988); Phys. Rev. B 392391 (1989).

[5] J. Suzuki, J. Phys. Soc. Jpn. 572966 (1988).

[6] R. Balian and G. Toulouse, Phys. Rev. Lett. 30544 (1973).

[7] M.E. Fisher, Phys. Rev. Lett. 30679 (1973).

[8] B. Duplantier and H. Saleur, Phys. Rev. Lett. 59539 (1987).

[9] H.W.J. Blöte, M.T. Batchelor and B. Nienhuis, Physica A 25195 (1998).

[10] H.W.J. Blöte and B. Nienhuis, J. Phys. A 221415 (1989).

[11] S.O. Warnaar, M.T. Batchelor and B. Nienhuis, J. Phys. A 253077 (1992).

[12] H.W.J. Blöte and M.P. Nightingale, Physica A 112405 (1982).

[13] M.P. Nightingale, Phys. Lett. A 59486 (1977); Proc. Kon. Ned. Ak. Wet. B 82235 (1979).

[14] J.L. Cardy, J. Phys. A 17 L385 (1984). 


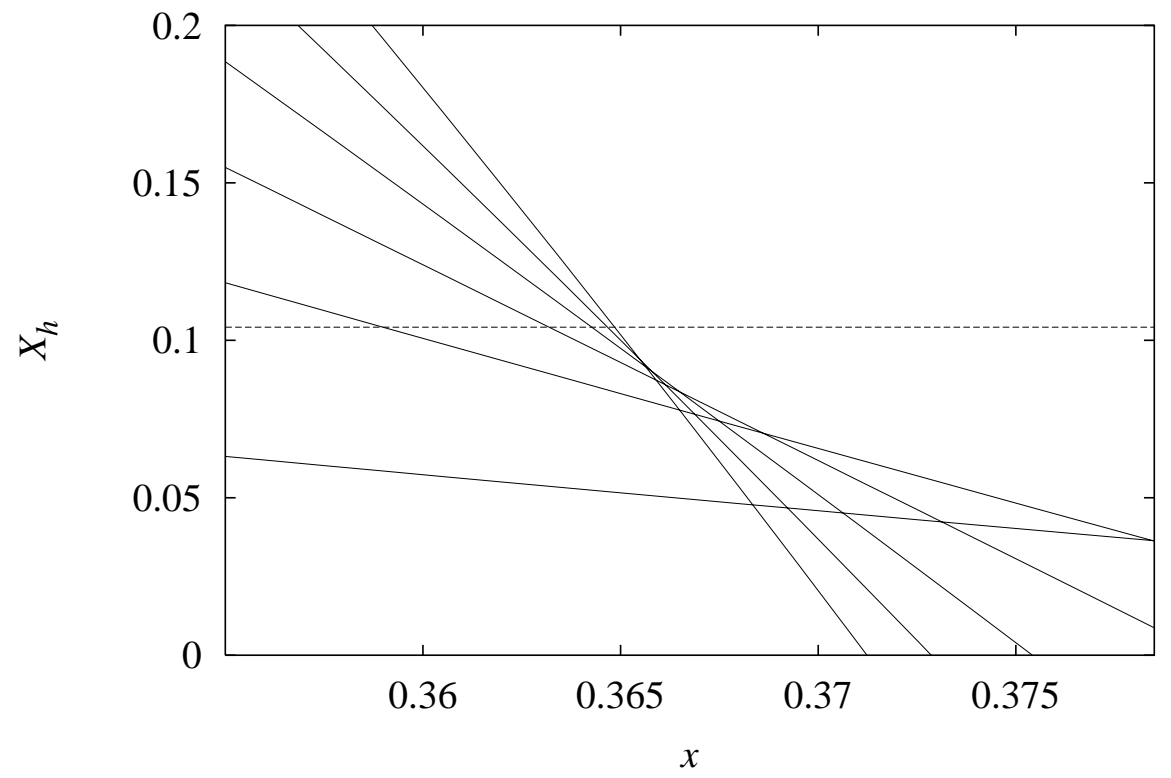

Figure 1: Scaled magnetic gap as a function of the bond weight $x$ at constant $z=0.3$. Different curves apply to different finite sizes $L=2,4,6,8,10$, and 12. The steeper the line, the larger $L$. The scaled gaps at the intersections are seen to approach the expected value $X_{h}=5 / 48$ (dotted line). 


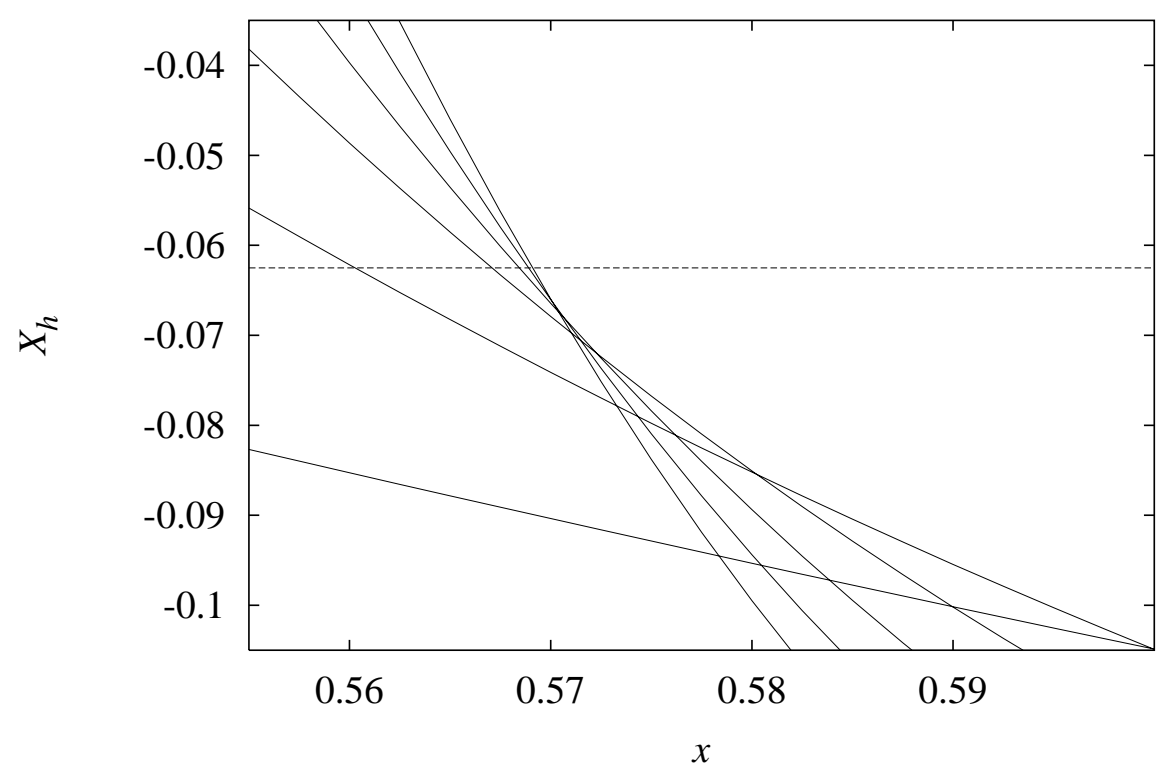

Figure 2: Scaled magnetic gap as a function of the bond weight $x$ at constant $z=0.8$. Different curves apply to different finite sizes $L=2,4,6,8,10$, and 12. The steeper the line, the larger $L$. The scaled gaps at the intersections are seen to approach the expected value $X_{h}=-1 / 16$ (dotted line). 


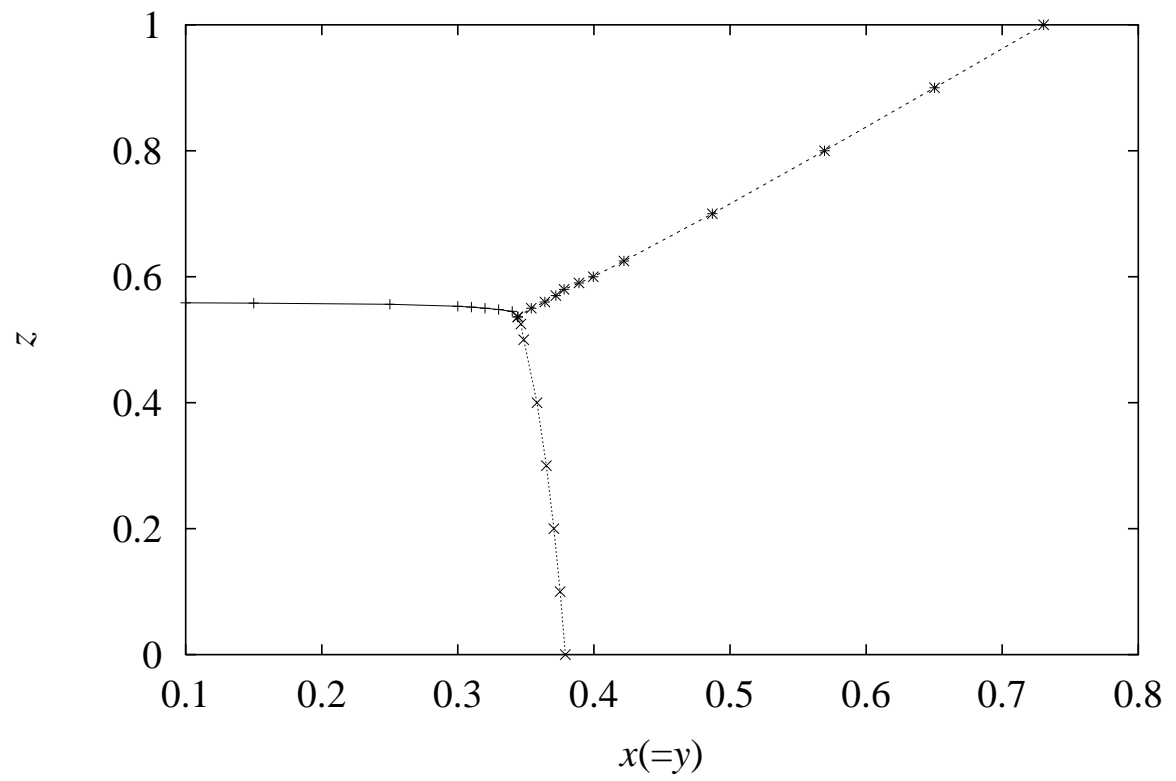

Figure 3: Phase diagram of the $\mathrm{O}(0)$ model for $x=y$. The vertical scale parametrizes the strength of the attractions between loop segments. The horizontal scale indicates the weight of vertices visited once by a loop. The data points were numerically obtained; their errors are much smaller than the symbol sizes. The curves serve only as a guide to the eye. 